УДК 681.7 .068

\author{
В.Д. Ефремов, А.А. Антропов, Д.С. Харенко
}

Институт автоматики и электрометрии СО РАН, Новосибирск, Россия

\title{
ПОДАВЛЕНИЕ ПЬЕДЕСТАЛА СВЕРХКОРОТКИХ ИМПУЛЬСОВ ПРИ ПОМОЩИ НЕЛИНЕЙНОГО УСИЛИВАЮЩЕГО ПЕТЛЕВОГО ЗЕРКАЛА
}

\begin{abstract}
Исследуются особенности прохождения ультракоротких импульсов с пьедесталом в спектральной области через нелинейное усиливающее петлевое зеркало. Построены карты максимального коэффициента пропускания, доли пьедестала в энергии импульса и изменения контраста в зависимости от длины петли и коэффициента усиления. Показано, что начальная энергия импульса, необходимая для достижения максимального коэффициента пропускания зеркала, падает ниже 1 нДж при длинах более 50 м и коэффициенте усиления более 5, а доля пьедестала может быть уменьшена с 34 до 5,6 \%.

Ключевые слова: волоконный фильтр, нелинейное усиливающее петлевое зеркало, численное моделирование, спектральный пьедестал, ультракороткие импульсы.
\end{abstract}

\section{V.D. Efremov, A.A. Antropov, D.S. Kharenko}

Institute of Automation and ElectrometrySB RAS, Novosibirsk, Russian Federation

\section{SUPPRESSION OF THE ULTRASHORT PULSE PEDESTAL USING A NONLINEAR AMPLIFYING LOOP MIRROR}

We have investigated the transmission of ultrashort pulses with a spectral pedestal in a nonlinear amplifying loop mirror. Maps of the maximum transmittance, the part of the pedestal in the pulse energy, and the change in contrast depending on the loop length and gain were presented. It has been shown that the initial energy required to achieve the maximum transmittance falls below $1 \mathrm{~nJ}$ for lengths over $50 \mathrm{~m}$ and a gain of more than 5, and the proportion of the pedestal can be reduced from 34 to 5.6 percent.

Keywords: fiber filter, nonlinear amplifying loop mirror, numerical simulation, spectral pedestal, ultrashort pulses.

\section{Введение}

Амплитудная самомодуляция нелинейного усиливающего петлевого зеркала (НУПЗ) позволяет использовать его как в качестве искусственного насыщающегося поглотителя в резонаторах с синхронизацией мод $[1,2]$, так и для амплитудной фильтрации импульсов с одновременным их усилением [3, 4]. Несмотря на множество способов генерации и многообразие лазерных схем, последняя функция остается по-прежнему востребованной, так как не всегда удается получать им- 
пульсы с подходящими для дальнейшего использования параметрами. Так, например, для CARS-спектроскопии необходимы импульсы с перестраиваемым узким спектром и высокой пиковой мощностью [5], от чего напрямую зависит разрешающая способность конечного прибора. Один из методов получения таких импульсов заключается в пропускании сильно чирпованных диссипативных солитонов через узкополосный фильтр [6]. В реальности у импульса остается некоторый пьедестал в спектральной области с амплитудой порядка $1 \%$ от пиковой мощности [7]. При последующем усилении значительная часть энергии переходит в подложку, спектр импульса расплывается, а необходимая пиковая мощность не достигается. Таким образом, существует необходимость подавить паразитное излучение для более эффективного последующего усиления.

В этой работе численно исследовалось, насколько НУПЗ подходит для задачи подавления спектрального пьедестала ультракоротких импульсов. Рассматривалась степень улучшения контраста при фиксированных длине петли и коэффициенте усиления в условиях максимального пропускания.

\section{Численное моделирование}

НУПЗ представляет собой два соединенных между собой выхода симметричного волоконного разветвителя. Вблизи одного из них расположен усилитель (рис. 1). Излучение низкой мощности, попадая в такую схему, отражается обратно в тот же порт. В случае сверхкоротких импульсов с высокой пиковой мощностью имеет место существенный нелинейный набег фазы. Излучение, распространяющееся в кольце по часовой стрелке, сначала испытывает усиление и затем проходит пассивное волокно. Из-за этого оно испытывает больший нелинейный набег фазы, чем излучение, распространяющееся против часов стрелки. Таким образом, результат интерференции на выходе в разветвителе зависит от таких параметров, как начальная пиковая мощность импульса, коэффициент усиления и длина пассивного волокна.

Основная сложность в подборе параметров НУПЗ под определенный импульс заключается в том, что оптимальная длина кольца зависит как от начальной энергии импульса, так и от коэффициента усиления. Фазовые набеги излучений, распространяющихся по часовой и против часовой стрелки, можно оценить по формулам соответственно [8]: 


$$
\delta \varphi_{c}=n_{2} g P_{0} L \pi / \lambda \text { и } \delta \varphi_{c c}=n_{2} P_{0} L \pi / \lambda
$$

где $n_{2}$ - нелинейный показатель преломления пассивного волокна, $g-$ коэффициент усиления, $P_{0}$ - пиковая мощность излучения и $L-$ длина пассивного волокна. Однако представленные формулы не описывают, как именно НУПЗ будет влиять на пьедестал в спектральной области. Поэтому для оценки параметров, необходимых и для успешного подавления пьедестала и для максимального пропускания импульса, целесообразно прибегнуть к численному моделированию.

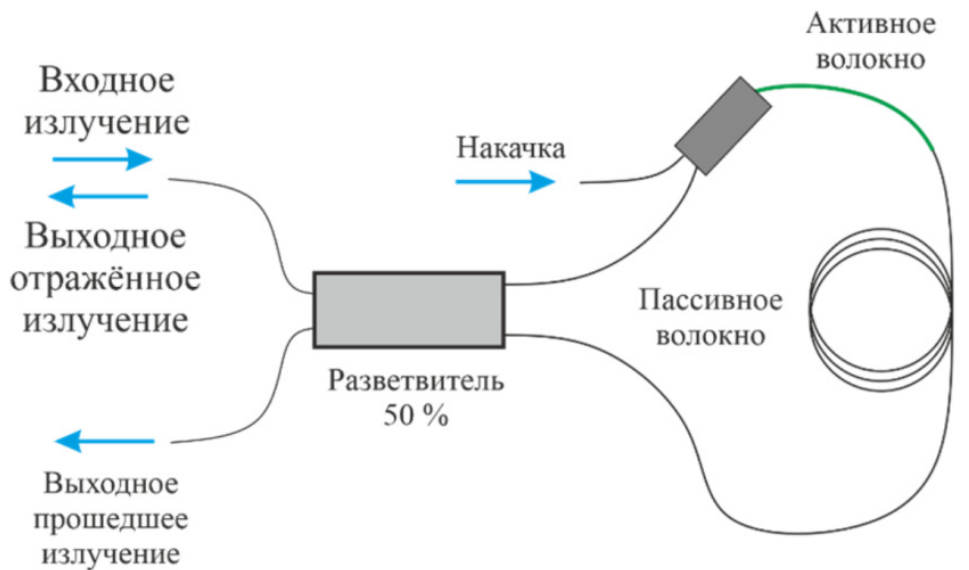

Рис. 1. Схема нелинейного усиливающего петлевого зеркала

Для моделирования НУПЗ использовалась численная модель на основе нелинейного уравнения Шредингера [9]:

$$
\pm \frac{\partial A}{\partial z}=i \gamma|A|^{2} A-\frac{i}{2} \beta_{2} \frac{\partial^{2} A}{\partial t^{2}}
$$

где $\gamma$ и $\beta_{2}$ - нелинейный и дисперсионный коэффициенты соответственно; \pm относится к излучениям, распространяющимся в кольце по часовой и против часовой стрелки; $A(z, t)$ - огибающая оптического поля. В данном случае импульсы распространяются в одном волокне в разных направлениях и, следовательно, не оказывают влияние друг на друга из-за крайне малого времени взаимодействия. Уравнения в системе (1) решаются независимо методом расщепления переменных по физическим процессам, в котором дисперсионный и нелинейный слагаемые 
решаются по отдельности на половине шага. Для реализации численной модели использовалась библиотека PyOFSS (Python-based optical fibre system simulator), написанная на языке программирования Рython [10]. Шаг по расстоянию варьировался от 400 до 5000 точек в зависимости от длины волокна. Временное окно составляло 4000 пс с количеством точек $2^{16}$. Для описания работы НУПЗ были добавлены такие модели элементов, как разветвитель 50/50 и несимметричный усилитель.

На рис. 2 представлена схема численной модели НУПЗ. Разветвитель, который разделяет входной импульс на два, задается матрицей (2) [11]:

$$
\left(\begin{array}{l}
A_{1}^{\prime} \\
A_{2}^{\prime}
\end{array}\right)=\left(\begin{array}{cc}
\frac{\sqrt{2}}{2} & i \frac{\sqrt{2}}{2} \\
i \frac{\sqrt{2}}{2} & \frac{\sqrt{2}}{2}
\end{array}\right)\left(\begin{array}{l}
A_{1} \\
A_{2}
\end{array}\right),
$$

где $A_{1}, A_{2}$ - излучение на входе в разветвитель, $A_{1}^{\prime}, A_{1}^{\prime}$ - излучение, получаемое на выходе. Усиление происходит точечно по формуле $A^{\prime}=g \cdot A$, где $g-$ фиксированный коэффициент точечного усиления. Насыщением было решено пренебречь и характеризовать усилитель лишь с точки зрения полного усиления сигнала. В качестве пассивного волокна использовалось стандартное одномодовое волокно с параметрами: $\gamma=6(\text { Вт } \cdot \text { км })^{-1}$ и $\beta_{2}=22 \mathrm{nc}^{2} \cdot \mathrm{\kappa м}^{-1}$.

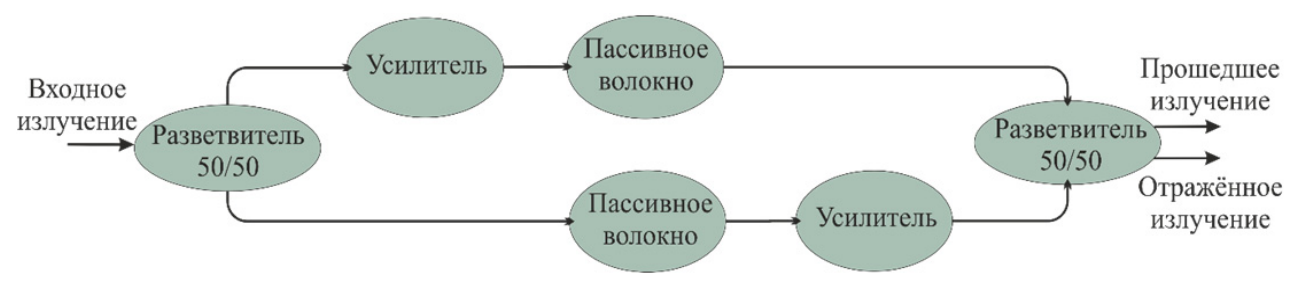

Рис. 2. Алгоритм распространения импульса в численной модели НУПЗ

Основным параметром, который используется для характеризации работы конкретной схемы НУПЗ, является коэффициент пропускания:

$$
T=\frac{E_{T}}{E_{T}+E_{R}}
$$


где $E_{T}$ и $E_{R}$ - энергии прошедшего и отраженного импульсов соответственно. На рис. 3 , $а$ показан характерный вид зависимости коэффициента пропускания $T$ от энергии входного импульса. Первый пик является максимально достижимым значением $T$, при котором наибольшая доля энергии проходит через НУПЗ. Конкретное значение $T_{\max }$ зависит от таких параметров, как $L$ - длина пассивного волокна в кольце, $g$ - коэффициент усиления и $E_{0}-$ энергия входного импульса.

Спектр модельного импульса до (пунктир) и после (сплошная линия) прохождения НУПЗ представлен на рис. 3, б. Ширина на полувысоте основной части составляет 78 пм, а пьедестала - 5,6 нм. Он содержит в себе $\sim 34$ \% энергии всего импульса. Длительность составляла 20 пс. Для нашей работы важно наиболее эффективно подавить спектральный пьедестал и одновременно с этим получить наибольший выход энергии из схемы. Поэтому был произведен поиск на плоскости параметров $L$ и $g$ таких $E_{0}$, при которых достигается $T_{\max }$. Для этого применялся следующий алгоритм. На вход в НУПЗ при некоторых фиксированных значениях $L$ ' и $g$ ' подавался импульс с фиксированной огибающей, энергия которого увеличивалась до тех пор, пока не достигался первый максимум $T_{\max }\left(L^{\prime}, g^{\prime}\right)$ (см. рис. $\left.3, a\right)$.

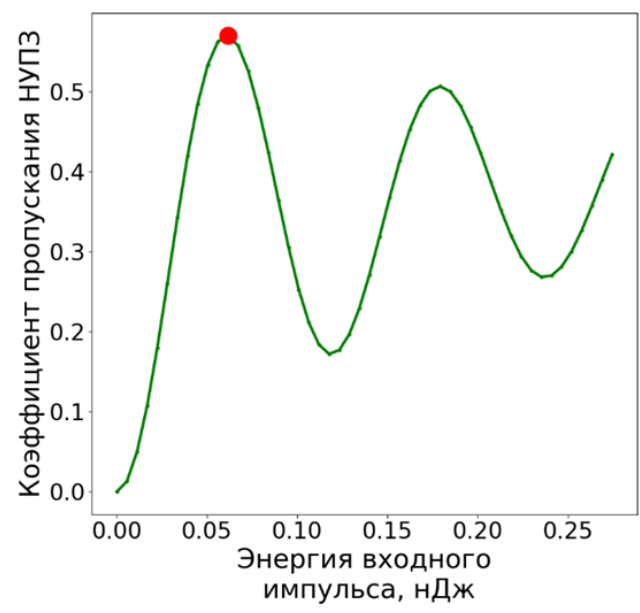

$a$

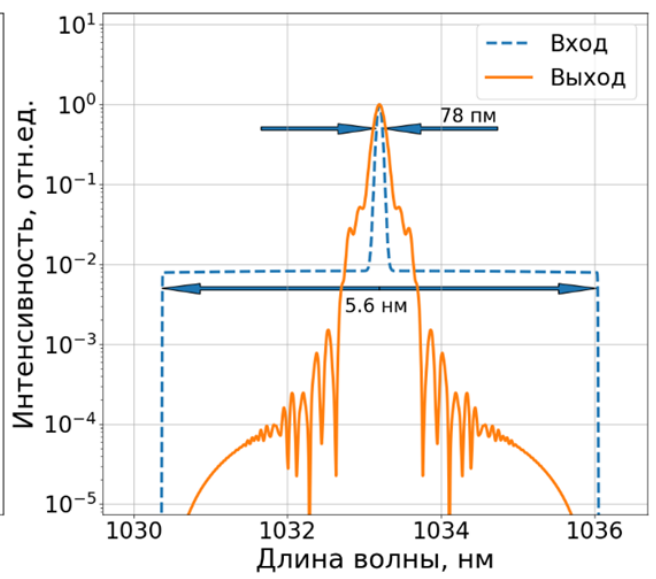

6

Рис. 3. Характерная для НУПЗ зависимость коэффициента пропускания от энергии входного импульса $(a)$ и нормированные спектры модельного импульса до и после прохождения пьедестала (б) 


\section{Результаты и обсуждение}

Таким образом, была составлена карта максимумов коэффициента пропускания $T_{\max }$ (рис. $4, a$ ), и далее в работе импульсы всегда исследовались в найденных точках. Длина пассивного волокна $L$ варьировалась от 1 м до 1 км, коэффициент усиления $g$ - от 2 до 20. В указанном диапазоне $T_{\max }$ варьировалось от 0,57 до 0,68 . На рис. $4, \sigma$ представлены энергии входного импульса, необходимые для достижения $T_{\max }$. Как хорошо видно, при малых длинах пассивного волокна и низком усилении требуются большие значения энергии (более 130 нДж). Однако при длинах более 50 м и коэффициенте усиления более 5 оптимальная начальная энергия падает ниже 1 нДж.

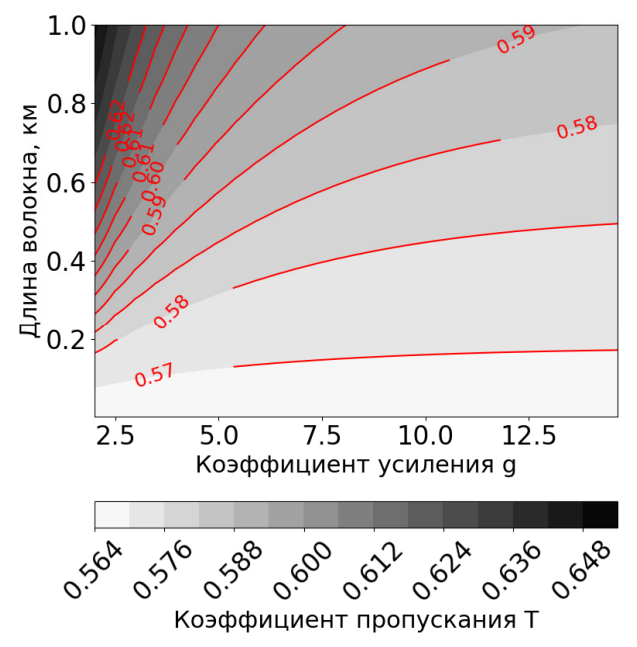

$a$

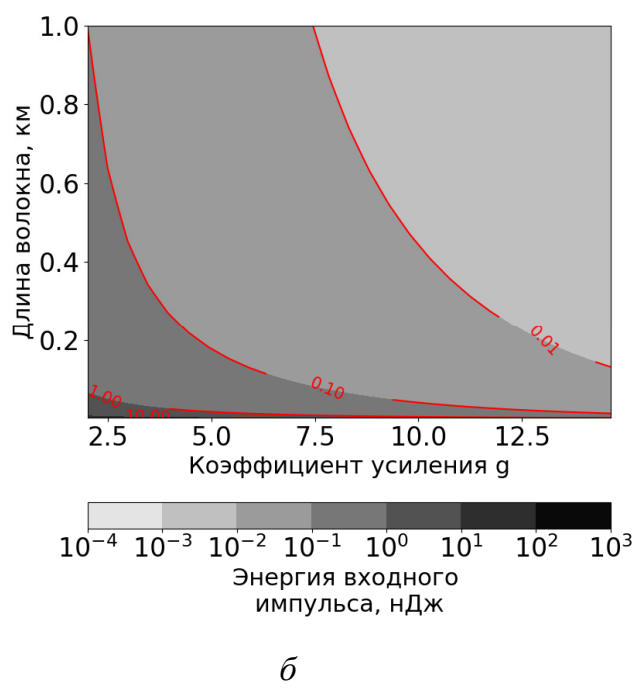

$\sigma$

Рис. 4. Карта максимумов коэффициента пропускания ( $a$ ) и энергия входного импульса, необходимая для достижения $T_{\max }$ (б)

На рис. 5, a представлена доля пьедестала от полной энергии прошедшего импульса, которая находилась путем моделирования прохождения через НУПЗ импульсов с такой же шириной спектра и длительностью, но без пьедестала. Сравнение энергий на выходе позволило оценить эффективность нелинейной фильтрации. С начальных $\sim 34 \%$ доля пьедестала уменьшалась до 5,6 \% в случае больших длин, порядка 1 км, и до 11,6 \% при малых длинах, порядка нескольких десятков метров. Все это свидетельствует о том, что пьедестал может 
быть эффективно подавлен при помощи НУПЗ. Однако стоит при этом рассмотреть влияние самой схемы на остальные параметры импульса. На рис. 5, б показана разница контрастов импульсов в спектральной области до и после прохождения НУПЗ в точках $T_{\max }$. Контраст начального импульса составлял 20,8 дБ. Хорошо видно, что разница контрастов в основном зависит от коэффициента усиления $g$. Причем при $g$ больше 5 (в зависимости от длины волокна) контраст импульса растет.
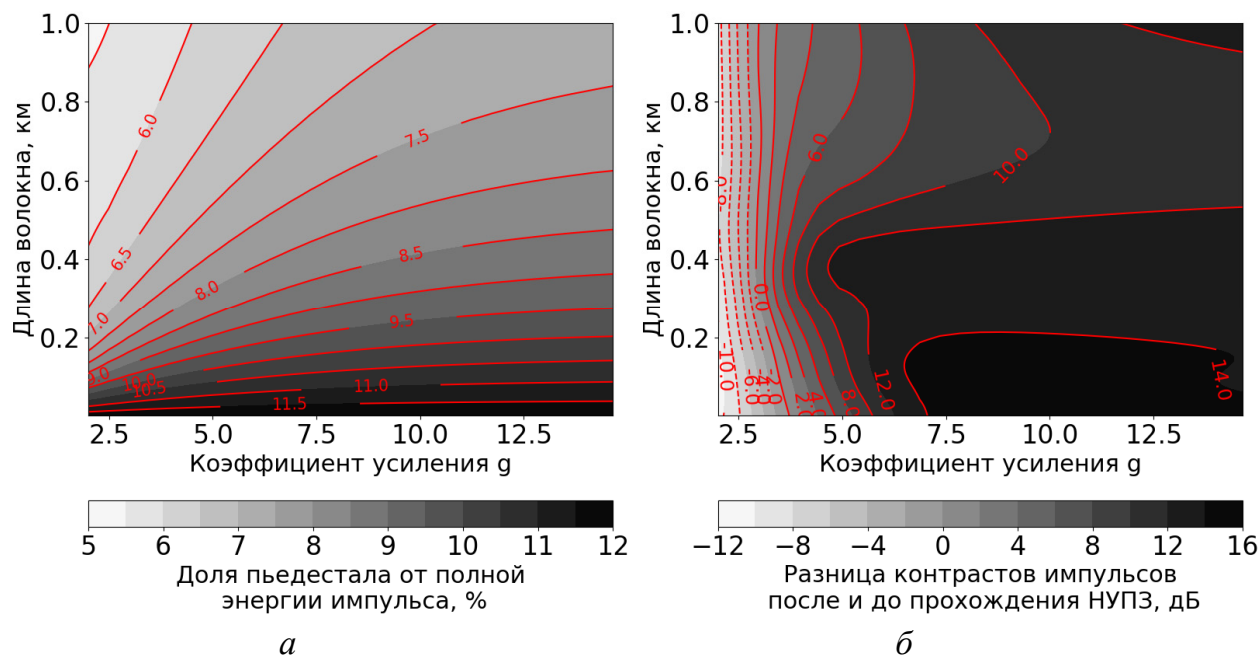

Рис. 5. Доля пьедестала от полной энергии импульса $(a)$ и разница контрастов импульсов после и до прохождения НУПЗ (б)

По представленным графикам (см. рис. 4, 5) можно сделать вывод, что наиболее перспективными параметрами НУПЗ для подавления пьедестала в спектральной области являются большие длины пассивного волокна и коэффициент усиления больше 5.

\section{Заключение}

В работе численно исследовалась возможность подавления спектрального пьедестала ультракоротких импульсов при помощи НУПЗ. В ходе моделирования были найдены максимальные значения коэффициента пропускания $T_{\max }$ на плоскости таких параметров, как длина пассивного волокна $L$ и коэффициент пропускания $g$. Было установлено, что $T_{\max }$ изменяется от 0,57 до 0,68 . Причем максимальные значе- 
ния достигаются при больших длинах волокна, близких к 1 км. Также была подсчитана доля пьедестала от полной энергии импульсов в точках $T_{\max }$ и показано, что она падает с начальных 34 до 5,6 \% при больших длинах пассивного волокна. С учетом вычисленной разницы контрастов импульсов в точках $T_{\max }$ до и после прохождения НУПЗ можно сделать вывод, что наиболее перспективными является область больших длин пассивного волокна и коэффициенты усиления больше 5, при которых выигрыш в контрасте после НУПЗ достигает 15 дБ.

Исследование выполнено при финансовой поддержке темы госзадания ИАиЭ СО РАН (рег. № АААA-А17-117062110026-3, Ефремов В.Д.) и РФФИ в рамках научного проекта № 20-32-70093 (Харенко Д.С., Антропов А.А.).

\section{Список литературы}

1. Mode-locked femtosecond all-normal all-PM Yb-doped fiber laser using a nonlinear amplifying loop mirror / C. Aguergaray, N.G.R. Broderick, M. Erkintalo, J.S.Y. Chen, V. Kruglov // Optics Express. - 2012. № 20. - P. 10545-10551.

2. Highly-stable mode-locked PM Yb-fiber laser with $10 \mathrm{~nJ}$ in 93-fs at $6 \mathrm{MHz}$ using NALM / Yang Yu, Hao Teng, Huibo Wang, Lina Wang, Jiangfeng Zhu, Shaobo Fang, Guoqing Chang, Junli Wang, Zhiyi Wei // Optics Express. - 2018. - № 26. - P. 10428-10434.

3. Smith K., Doran N.J., Wigley P.G.J. Pulse shaping, compression, and pedestal suppression employing a nonlinear-optical loop mirror // Optics Letters. - 1990. - № 15. - P. 1294-1296.

4. Dajun Lei, Xiquan Fu, Shuangchun Wen. Effect of gain bandwidth on the amplification of ultrabroad bandwidth pulse in an erbium-doped nonlinear amplifying fibre loop mirror // Opt. A: Pure Appl. Opt. - 2007. № 9. - P.114-121.

5. Nonlinear microscopy, infrared, and Raman microspectroscopy for brain tumor analysis / T. Meyer, N. Bergner, C. Krafft, D. Akimov, B. Dietzek, J. Popp, C. Bielecki, B.F.M. Romeike, R. Reichart, R. Kalff // J. of Biomedical Optics. - 2011. - № 16. - P. 021113.

6. Fiber-based source for multiplex-CARS microscopy based on degenerate four-wave mixing / Thomas Gottschall, Martin Baumgartl, Aude 
Sagnier, Jan Rothhardt, Cesar Jauregui, Jens Limpert, Andreas Tünnermann // Optics Express. - 2012. - Vol. 20.

7. Development of robust fiber laser source based on parametric frequency conversion for use in CARS microscopy / E.A. Evmenova, A.A. Antropov, D.S. Kharenko, A.G. Kuznetsov, S.I. Kablukov, S.A. Babin // Optics in Health Care and Biomedical Optics IX. "SPIE/COS Photonics Asia". - Hangzhou, China: Proceedings SPIE. - 2019. - Vol. 11190.

8. Pearson G.W., Zanoni R., Krasinski J.S. Analysis of ultra-short pulse propagation in a fiber nonlinear amplifying loop mirror // Optics Communications. - 1993. - № 103. - P. 507-518.

9. Properties of artificial saturable absorbers based on NALM with two pumped active fibres / A. Kokhanovskiy, S. Kobtsev, A. Ivanenko, S. Smirnov // Laser Physics Letters. - 2018. - № 15. - P. 125101.

10. GitHub. - URL: https:/github.com/galilley/pyofss/ (дата обращения: 01.10.2020).

11. Govind P. Agrawal. Applications of Nonlinear Fiber Optics. Academic Press, 2008.

\section{References}

1. Aguergaray C., Broderick N.G.R., Erkintalo M., Chen J.S.Y., Kruglov V. Mode-locked femtosecond all-normal all-PM Yb-doped fiber laser using a nonlinear amplifying loop mirror. Optics Express, 2012, no. 20, pp. 10545-10551.

2. Yu Yang, Teng Hao, Wang Huibo, Wang Lina, Zhu Jiangfeng, Fang Shaobo, Chang Guoqing, Wang Junli, Wei Zhiyi. Highly-stable modelocked PM Yb-fiber laser with $10 \mathrm{~nJ}$ in 93-fs at $6 \mathrm{MHz}$ using NALM. Optics Express, 2018, no. 26, pp. 10428-10434.

3. Smith K., Doran N.J., Wigley P.G.J. Pulse shaping, compression, and pedestal suppression employing a nonlinear-optical loop mirror. Optics Letters, 1990, no. 15, pp. 1294-1296.

4. Lei Dajun, Fu Xiquan, Wen Shuangchun. Effect of gain bandwidth on the amplification of ultrabroad bandwidth pulse in an erbium-doped nonlinear amplifying fibre loop mirror. Opt. A: Pure Appl. Opt., 2007, no. 9, pp. 114-121.

5. Meyer T., Bergner N., Krafft C., Akimov D., Dietzek B., Popp J., Bielecki C., Romeike B.F.M., Reichart R., Kalff R. Nonlinear microscopy, 
infrared, and Raman microspectroscopy for brain tumor analysis. J. of Biomedical Optics, 2011, no. 16, 021113 p.

6. Gottschall Thomas, Baumgartl Martin, Sagnier Aude, Rothhardt Jan, Jauregui Cesar, Limpert Jens, Tünnermann Andreas. Fiber-based source for multiplex-CARS microscopy based on degenerate four-wave mixing. Optics Express, 2012, vol. 20.

7. Evmenova E.A., Antropov A.A., Kharenko D.S., Kuznetsov A.G., Kablukov S.I., Babin S.A. Development of robust fiber laser source based on parametric frequency conversion for use in CARS microscopy. Optics in Health Care and Biomedical Optics IX. "SPIE/COS PHOTONICS ASIA". Hangzhou, China: Proceedings SPIE, 2019, vol. 11190.

8. Pearson G.W., Zanoni R., Krasinski J.S. Analysis of ultra-short pulse propagation in a fiber nonlinear amplifying loop mirror. Optics Communications, 1993, no. 103, pp. 507-518.

9. Kokhanovskiy A., Kobtsev S., Ivanenko A., Smirnov S. Properties of artificial saturable absorbers based on NALM with two pumped active fibres. Laser Physics Letters, 2018, no. 15, 125101 p.

10. GitHub, available at: https://github.com/galilley/pyofss/ (accessed 01 October 2020).

11. Govind P. Agrawal. Applications of Nonlinear Fiber Optics. Academic Press, 2008. 